\title{
Successful nonsurgical treatment of tooth with periapical lesions using bioceramic sealer: a case report
}

\author{
Gyulbenkiyan Elvira ${ }^{1}$, Gusiyska Angela $^{1}$
}

1. Department of Conservative Dentistry, Faculty of Dental Medicine, Medical University - Sofia, Bulgaria;

\begin{abstract}
The aim of this case report is to describe a mandibular first molar with large periapical lesions, which after shaping and disinfection of the root canals obturation with bioceramic sealer was done. A 12-month follow-up was done using radiographic examination as the analysis of the periapical reduction was evaluated with Periapical Index (PAI) by Ǿrstavik. For a year the periapical lesion reduced from PAI 5 to $P A I 2$ as the tooth remains asymptomatic.
\end{abstract}

Keywords: apical periodontitis, retreatment, bioceramic sealer, osteogenesis

\section{Introduction}

The chronic apical periodontitis is a nonspecific inflammatory process of both dental and periapical tissues, caused by a variety of microorganisms that activate an immunological inflammatory response in the macroorganism $(1,2)$. The clinical and radiographic success of root canal treatment depends on the complete elimination of microorganisms from the root canal system and the prevention of reinfection. One of the main functions of the root canal sealers are sealing voids, accessory canals, and multiple foramina, bonding the core filling material with the root canal walls, and entombing any remaining bacteria (3). Bioceramics are ceramic materials designed for medical and dental use. They are inorganic, non-metallic, 
biocompatible and include elements such as aluminum, zirconium, bioactive glass, glass ceramic particles, hydroxylapatite, and calcium phosphate $(4,5,6)$. The bioceramics are chemically stable, non-corrosive, and interact well with organic tissue (7).

Total Fill BC (FKG, Switzerland) is a pre-mixed, hydrophilic bioceramic sealer consisting of calcium silicate, calcium hydroxide, calcium phosphate and zirconium oxide. The sealer is radiopaque, and aluminum free with a high $\mathrm{pH}$, and require moisture to set and harden. During setting, it forms hydroxylapatite crystals through which it chemically binds to dentin. The sealer is used with a monocone technique using calibrated gutta-percha points (Total Fill BC Points) coated with nanobioceramic particles, thus creating a chemical bond between the sealer and the gutta-percha. As a result, a tertiary monoblock is realized, which increases the resistance of the root canal $(7,8)$.

\section{Case report}

A 37 year old female was referred for endodontic treatment with pain in relation to her mandibular right first molar (46). The tooth on examination revealed a crown destruction. The patient had pain on percussion. Intra oral radiograph revealed a tooth with not exacting endodontic treatment with separate endodontic instruments in mesial lingual (ML), and in distal (D) root canals, and with particularly large apical radiolucency (figure 1).

Diagnosis: previously treated, symptomatic apical periodontitis

Treatment is nonsurgical endodontic retreatment followed by permanent restoration of the access cavity, and crown restoration.

Under rubber dam isolation an access cavity was prepared. Three orifices were located on the floor of the pulp chamber. The retreatment was done using crown-down technique ( $\mathrm{H}$-files, Dentsply, Maillefer, Switzerland). The separate instruments were pull out using ultrasonic. $\mathrm{Ca}(\mathrm{OH}) 2$ as inter-appointment intracanal medicament was applied during the endodontic treatment for 10 days. Cleaning and shaping was realized by rotary instrumentation ProTaper Universal \#40, (Dentsply, Maillefer, Switzerland). A thorough irrigation with sodium hypochlorite $2.5 \%$ and saline was done after each instrument throughout the procedure. The final disinfection performed after the root canal preparation was finished with subsequent irrigation with $\mathrm{NaOCl} 2.5 \%$, EDTA 15\% and saline. All root canals were obturated with bioceramic sealer Total Fill BC (FKG, Switzerland) using monocone technique with calibrated gutta-percha points (Total Fill BC Points, FKG, Switzerland) (figure 2). Bioceramic sealer was extruded periapical. 


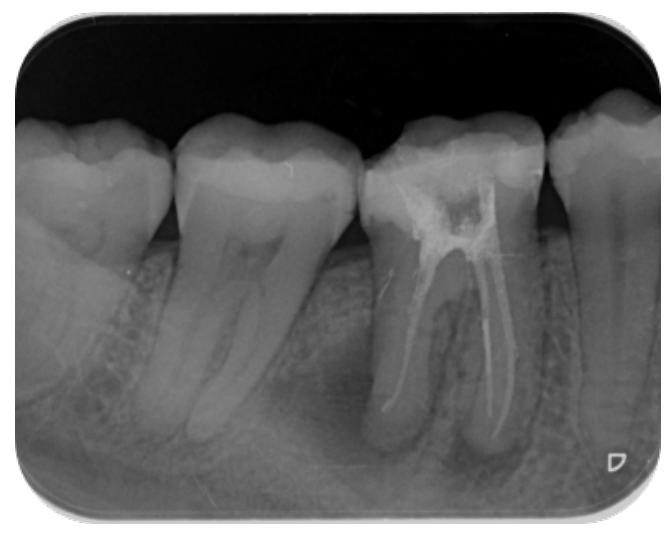

Fig. 1. Radiograph of tooth 46 before treatment (PAI 5)

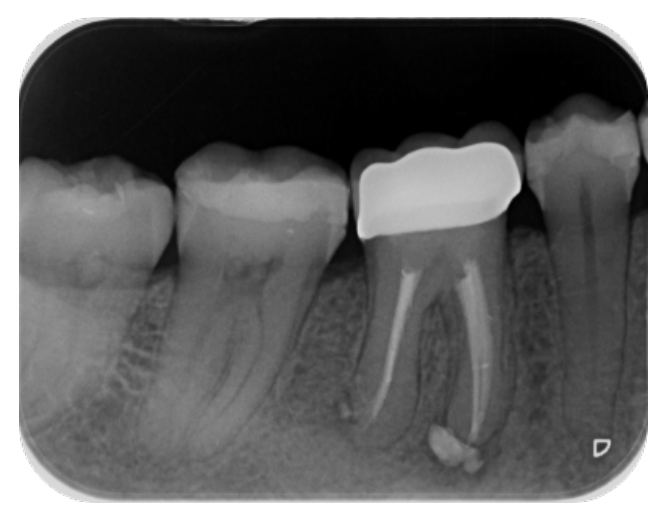

Fig. 3. 6th-month follow-up of tooth 46 (PAI 3)

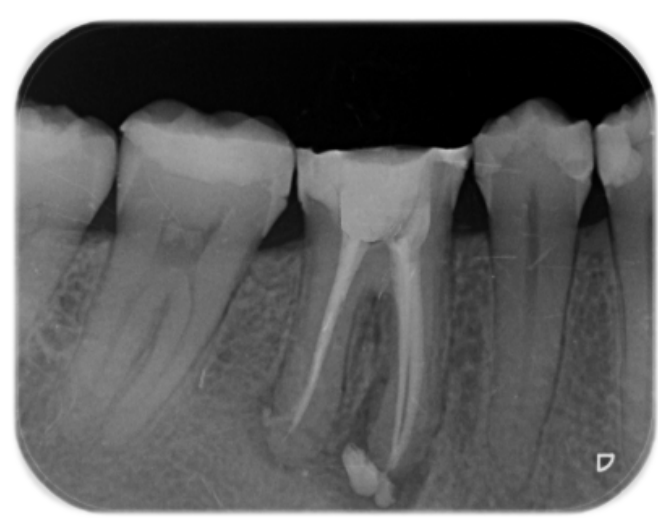

Fig. 2. Radiograph of tooth 46 during treatment (PAI 5)

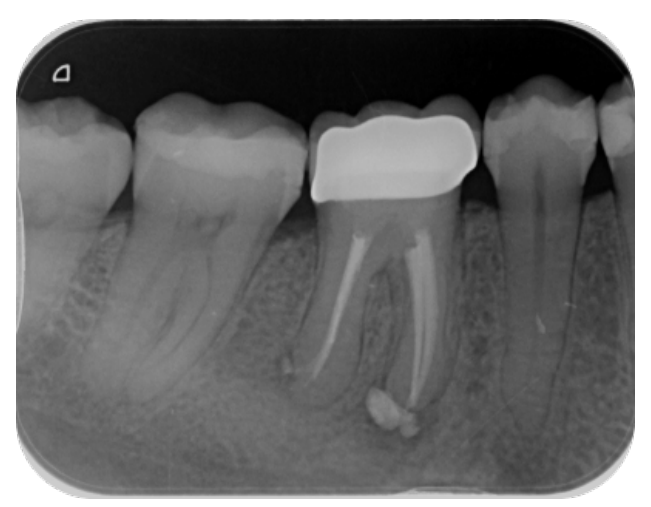

Fig. 4. 12th-month follow-up of tooth 46 (PAI 2)

A 12-month follow-up was done using radiographic examination (figures 3,4 ), as the analysis of the periapical reduction was evaluated with Periapical Index (PAI) by Ǿrstavik (9) (table 1). For a year the periapical lesion reduced from PAI 5 to PAI 2 as the tooth remains asymptomatic. The prosthetic treatment with zirconium crown protects the root from fracture. 
Tab. 1. Periapical Index (Ǿstravik et al.)

\section{PAI Score Description of Radiographic findings}

$\begin{array}{ll}\mathbf{1} & \text { Normal Periapical Structures } \\ \mathbf{2} & \text { Small changes in Bone Structures } \\ \mathbf{3} & \text { Change in bone Structures with Mineral Loss } \\ \mathbf{4} & \text { Periodontitis with well-defined radiolucent area } \\ \mathbf{5} & \text { Severe periodontitis with exacerbating features }\end{array}$

\section{Discussion}

With both antimicrobial and sealing properties, premixed bioceramics are unique materials available in endodontics that contribute to the success of both the radiographic and clinical outcome. The bioceramic sealer Total Fill BC has the advantage of being highly bio-tolerable to surrounding tissues due to its chemical and crystalline structure close to that of dental tissues and bone $(10,11)$. This case presents the outcome of regenerative periapical endodontic treatment with bioceramic sealer applied on tooth clinically defined as having hopeless prognosis as a consequence of the presence of a chronic periodontal lesions evaluated with PAI 5. Due to the resorption of the root apices the sealer was extruded periapical during the obturation procedure (figure 2). Kossev and Stefanov (12) found that after root canal obturation with bioceramic sealers by extruding material into the periapical area, there was extremely little or no postoperative sensitivity. The osteoconductive and osteoinductive ability of the material stimulates the osteogenesis by activating the osteoblast cells. For 12-months follow-up the periapical lesion reduced from PAI 5 to PAI 2 (figure 4) without any resorption of the extruded bioceramic material as we supposed that a longer period is required for complete mineralization and healing of the periodontium in the apical area.

The biocompatibility and biomineralization effects of Total Fill BC reveal promising results as bioceramic root canal filling material.

\section{Conclusion}

The regenerative, antibacterial and sealing ability of bioceramic sealers demonstrates promising and successful endodontic outcome in teeth with periapical lesions. 


\section{References}

1. Pihlstrom B.L., B. S. Michalowicz, N. W. Johnson. Periodontal diseases. Lancet, 2005; 366 (9499), 1809-1820.

2. Silva N., L. Abusleme, D. Bravo, N. Dutzan, J. Garcia-Sesnich, R. Vernal, M. Hernandez, J. Gamonal. Host response mechanisms in periodontal diseases. J. Appl. Oral Sci., 2015; 23 (3) : 329-355.

3. Kaur A., N. Shah, A. Logani, N. Mishra, "Biotoxicity of commonly used root canal sealers: a meta-analysis," Journal of Conservative Dentistry, 2015; 18 (2) : 83-88.

4. Best S.M., A. E. Porter, E.S. Thian, J.Huang. Bioceramics: past, present and for the future. J Eur Ceram Soc, 2008;28:1319-1327.

5. Dubok V. A. Bioceramics: yesterday, today, tomorrow. Powder Metall Metal Ceram 2000;39:381-394.

6. Hench L.L. Bioceramics: from concept to clinic. J Am Ceram Soc 1991;74:1487-1510.

7. Debelian G, M. Trope. The use of premixed bioceramic materials in endodontics. Giornale Italiano di Endodonzia, 2016 Nov, http://dx.doi.org/10.1016/j.gien.2016.09.001.

8. Yang S. E., S. H. Baek, W. Lee, et al, "In vitro evaluation of the sealing ability of newly developed calciumphosphate-based root canal sealer," Journal of Endodontics, 2007; 33, pp. 978-981.

9. Ørstavik D., K. Kerekes, H. M. Eriksen. The periapical index: a scoring system for radiographic assessment of apical periodontitis. Endod Dent Traumatol. 1986; Feb;2(1):20-34.

10. Ginebra M. P., E. Fern'andez, E. A. P. De Maeyer et al. Setting reaction and hardening of an apatitic calcium phosphate cement. Journal of Dental Research, 1997; 76 (4),905-912.

11. Koch K., D. Brave. Anewday has dawned: the increased use of bioceramics in endodontics. Dentaltown, 2009; vol. 10, 39-43.

12. Kossev D., V. Stefanov. Ceramics-based sealers as new alternative to currently used endodontic sealers. research ceramics-based sealers, 2009; 1:42-48.

\section{Corresponding author:}

Elvira Gyulbenkiyan,

Department of Conservative Dentistry and Endodontics,

Faculty of Dental Medicine,

Medical University-Sofia, Bulgaria,

1 Georgy Sofiyski Str.

tel: +359898348535

email: elvira.simon@gmail.com 